\title{
The impact of aspirin and anticoagulant usage on outcomes after aneurysmal subarachnoid hemorrhage: a Nationwide Inpatient Sample analysis
}

\author{
Hormuzdiyar H. Dasenbrock, MD, ${ }^{1-4}$ Sandra C. Yan, BS, BA, ${ }^{1,2,5}$ Bradley A. Gross, MD, ${ }^{6}$ \\ Donovan Guttieres, ${ }^{2}$ William B. Gormley, MD, MPH, ${ }^{1-3}$ Kai U. Frerichs, MD, ${ }^{1-3}$ \\ M. Ali Aziz-Sultan, MD, ${ }^{1-3}$ and Rose Du, MD, PhD'-3 \\ ${ }^{1}$ Cushing Neurosurgical Outcomes Center, ${ }^{2}$ Department of Neurological Surgery, Brigham and Women's Hospital, \\ ${ }^{3}$ Harvard Medical School, and ${ }^{4}$ H.T. Chan Harvard School of Public Health, Boston, Massachusetts; ${ }^{5}$ Warren Alpert School \\ of Medicine, Brown University, Providence, Rhode Island; and ${ }^{6}$ Barrow Neurological Institute, Division of Neurological \\ Surgery, Phoenix, Arizona
}

OBJECTIVE Although aspirin usage may be associated with a decreased risk of rupture of cerebral aneurysms, any potential therapeutic benefit from aspirin must be weighed against the theoretical risk of greater hemorrhage volume if subarachnoid hemorrhage (SAH) occurs. However, few studies have evaluated the association between prehemorrhage aspirin use and outcomes. This is the first nationwide analysis to evaluate the impact of long-term aspirin and anticoagulant use on outcomes after SAH.

METHODS Data from the Nationwide Inpatient Sample (NIS; 2006-2011) were extracted. Patients with a primary diagnosis of SAH who underwent microsurgical or endovascular aneurysm repair were included; those with a diagnosis of an arteriovenous malformation were excluded. Multivariable logistic regression was performed to calculate the adjusted odds of in-hospital mortality, a nonroutine discharge (any discharge other than to home), or a poor outcome (death, discharge to institutional care, tracheostomy, or gastrostomy) for patients with long-term aspirin or anticoagulant use. Multivariable linear regression was used to evaluate length of hospital stay. Covariates included patient age, sex, comorbidities, primary payer, NIS-SAH severity scale, intracerebral hemorrhage, cerebral edema, herniation, modality of aneurysm repair, hospital bed size, and whether the hospital was a teaching hospital. Subgroup analyses exclusively evaluated patients treated surgically or endovascularly.

RESULTS The study examined 11,549 hospital admissions. Both aspirin $(2.1 \%, n=245)$ and anticoagulant users $(0.9 \%$, $n=108$ ) were significantly older and had a greater burden of comorbid disease $(p<0.001)$; severity of SAH was slightly lower in those with long-term aspirin use $(p=0.03)$. Neither in-hospital mortality $(13.5 \%$ vs $12.6 \%)$ nor total complication rates $(79.6 \%$ vs $80.0 \%)$ differed significantly by long-term aspirin use. Additionally, aspirin use was associated with decreased odds of a cardiac complication (OR $0.57,95 \% \mathrm{Cl} 0.36 \%-0.91 \%, p=0.02$ ) or of venous thromboembolic events (OR $0.53,95 \% \mathrm{Cl} 0.30 \%-0.94 \%, p=0.03$ ). Length of stay was significantly shorter (15 days vs 17 days [12.73\%], 95\% $\mathrm{Cl} 5.22 \%-20.24 \%, p=0.001)$, and the odds of a nonroutine discharge were lower (OR $0.63,95 \% \mathrm{Cl} 0.48 \%-0.83 \%, p=$ 0.001 ) for aspirin users. In subgroup analyses, the benefits of aspirin were primarily noted in patients who underwent coil embolization; likewise, among patients treated endovascularly, the adjusted odds of a poor outcome were lower among long-term aspirin users (31.8\% vs $37.4 \%$, OR $0.63,95 \% \mathrm{Cl} 0.42 \%-0.94 \%, \mathrm{p}=0.03)$. Although the crude rates of inhospital mortality (19.4\% vs $12.6 \%$ ) and poor outcome (53.6\% vs $37.6 \%$ ) were higher for long-term anticoagulant users, in multivariable logistic regression models these variations were not significantly different (mortality: OR $1.36,95 \% \mathrm{Cl}$ $0.89 \%-2.07 \%, p=0.16$; poor outcome: OR 1.09, 95\% Cl 0.69\%-1.73\%, $p=0.72$ ).

CONCLUSIONS In this nationwide study, neither long-term aspirin nor anticoagulant use were associated with differential mortality or complication rates after SAH. Aspirin use was associated with a shorter hospital stay and lower rates of nonroutine discharge, with these benefits primarily observed in patients treated endovascularly.

http://thejns.org/doi/abs/10.3171/2015.12.JNS151107

KEY WORDS acetylsalicylic acid; aspirin; anticoagulant; anticoagulation; cerebral aneurysm; endovascular; subarachnoid hemorrhage; vascular disorders

ABBREVIATIONS CI = confidence interval; COX = cyclooxygenase inhibitor; ICD-9-CM = International Classification of Diseases, 9th Revision, Clinical Modification; IQR = interquartile range; ISUIA = International Study of Unruptured Intracranial Aneurysms; mRS = modified Rankin Scale; NIS = Nationwide Inpatient Sample; NIS-SAH SS = NIS-SAH severity scale; NIS-SOM = NIS-SAH outcome measure; SAH = subarachnoid hemorrhage.

SUBMITTED May 12, 2015. ACCEPTED December 29, 2015.

INCLUDE WHEN CITING Published online April 8, 2016; DOI: 10.3171/2015.12.JNS151107 
A SPIRIN is one of the most commonly used medications internationally. Its wide dissemination, low cost, acceptable safety profile, and daily dosing make it an excellent therapeutic agent for public health interventions. In addition to its use as an analgesic, aspirin reduces the risk of cardiovascular ${ }^{5,22,37}$ and cerebrovascular disease..$^{32,34,45}$ Recent data have also suggested that aspirin may decrease the risk of rupture of cerebral aneurysms. ${ }^{20,21}$ Inflammatory pathways promote aneurysm formation. ${ }^{9,16,17}$ Thus, it is logical that antiinflammatory medications may be protective against the development of and hemorrhage from cerebral aneurysms. In a retrospective analysis of the International Study of Unruptured Intracranial Aneurysms (ISUIA), Hasan et al. reported that aspirin use at least 3 times weekly was associated with lower adjusted odds of aneurysmal subarachnoid hemorrhage $(\mathrm{SAH}){ }^{21}$

Nonetheless, any potential therapeutic benefits from the antiinflammatory properties of aspirin must be weighed against the theoretical risk of greater hemorrhage volume from the antithrombotic properties of aspirin if rupture of a given aneurysm were to occur. Addressing this is critical if aspirin is to be recommended to patients with known cerebral aneurysms: enthusiasm for using aspirin as an agent for secondary prevention will be limited if those in whom SAH occurs while on aspirin have inferior outcomes. Two retrospective institutional reviews reported that premorbid aspirin use was not associated with differential outcomes after SAH..$^{20,39}$ However, the sample sizes of these studies were 305 and 274 patients, potentially making them underpowered to detect subtle-yet clinically pertinent-differences in outcomes.

Given the importance of understanding the relationship between prehemorrhage aspirin use and outcomes after $\mathrm{SAH}$, as well as the potential for institutional reviews to be underpowered to assess this relationship, evaluation of this association with a nationwide data source is merited. The goal of this study was to use the Nationwide Inpatient Sample (NIS) to evaluate to what degree the in-hospital outcomes after aneurysmal SAH vary based on long-term aspirin and anticoagulant usage.

\section{Methods \\ Data Source}

Data were extracted from the NIS (Healthcare Cost and Utilization Project, Agency Healthcare Research and Quality) from 2006 to 2011. The year 2006 was the first full year that the International Classification of Diseases, 9th Revision, Clinical Modification (ICD-9-CM) diagnosis code for long-term aspirin use was used. The NIS, a $20 \%$ stratified sample of nonfederal hospitals, is the largest all-payer national database in the $\mathrm{US}^{2}$ and has previously been used to evaluate the outcomes of patients with aneurysmal SAH. 1,3,4,6,10,11,15,23-26,30,31,36,43,44 Our institutional review board deemed that studies utilizing the NIS are exempt and not classified as human subjects research.

\section{Inclusion Criteria and Patient Stratification}

Patients were included if they had ICD-9-CM diagnosis codes of SAH (430) or intracranial hemorrhage (431,
432.9); underwent aneurysm repair by microsurgical clipping (39.51) or endovascular embolization (39.72, 39.75, $39.76,39.79)$; and were at least 18 years of age. Only patients with a nonelective admission were included. Admissions with a diagnosis code for a cerebrovascular malformation (747.81), syphilitic aneurysm (094.87), and cerebral arteritis (437.4), as well as with a procedure code for arteriovenous malformation repair (39.53) or stereotactic radiosurgery (923.x) were excluded. Patients were stratified based on the ICD-9-CM diagnosis codes for long-term aspirin use (V58.66) and long-term anticoagulant use (V58.61).

\section{Covariates}

Patient age and sex were extracted from the NIS database. Comorbidities were analyzed using the Elixhauser et al. index. ${ }^{14}$ Neurological deficits, paralysis, and electrolyte complications were not included in the comorbidity score, given the potential misclassification with SAH and its associated complications. Comorbidities present in 10 or fewer patients (lymphoma and hemorrhagic peptic ulcer) were also excluded from the total score. Atrial fibrillation is an important indication for anticoagulation, but is not included in the Elixhauser comorbidity index. Therefore, atrial fibrillation was extracted (427.21) and used as a covariate in analyses of anticoagulation. Insurance status, hospital bed size, and whether the hospital was a teaching hospital are encoded in the NIS. Hospital bed size and teaching status could affect the quality of care.

Clinical data available in the NIS are encoded through ICD-9-CM codes; therefore, pertinent endpoints without corresponding ICD-9-CM codes (such as Hunt and Hess or Fisher grade) could not be analyzed. However, the NISSAH severity scale (NIS-SAH SS), a validated severity adjustment scale with good concordance with Hunt and Hess grade, was calculated for each patient based on the diagnosis codes for coma, hydrocephalus, hemiparesis, aphasia, and cranial nerve deficits, as well as procedure codes for CSF diversion procedures and mechanical ventilation in accordance with the published methodology. ${ }^{44}$ Additionally, the proportion of patients who presented with intraparenchymal or intraventricular hemorrhage (431) was analyzed due to its association with higher Fisher grade. The treatment modality used for aneurysm repair was also used as a covariate to partially account for variations in institutional practice and aneurysm location. The proportion of patients who underwent decompressive craniectomy (01.25) and who had a diagnosis code for cerebral herniation (348.4) or cerebral edema (348.5) were extracted to partially account for elevated intracranial pressure. Platelet (99.05) and plasma (99.07) transfusions were also analyzed.

\section{Outcomes}

Outcomes evaluated included in-hospital mortality and length of hospital stay; additionally, complications were extracted. Total complications included neurological (seizures [345.xx], stroke [433.x and 434.x], transient cerebral ischemia [435.x], and neurological complications after procedure [997.01 and 997.09]), cardiac (410.xx, 248. $\mathrm{xx}, 427.5,785 . x x)$, pulmonary (514.x, 518.xx, 512.x), renal (584.x), gastrointestinal (578.x, 5601, 00845), venous 
thromboembolic (453.x, 415.x), hematological (285.x and 998.1x, as well as red blood cell transfusion 94.04), sodium disturbances $(253.5,253.6,276.0,276.1)$, and infectious complications (595.0, 996.64, 481-486, 507.0, 997.31, 38.x, 995.9x, 320.x, 041.x, 324.1, 790.7, 999.31, and 998.59). Total complication rate, neurological, cardiac, and hematological complications, and venous thromboembolic events were evaluated as different outcomes given the potential relationship between these complications and antithrombotic medication usage. The proportion of patients who underwent tracheostomy $(311,31.21,31.29)$ and gastrostomy or jejunostomy $(43.11,43.19,46.32)$ was evaluated. A nonroutine discharge was defined as any discharge other than to home. Analyses of length of stay, placement of tracheostomy or gastrostomy, and discharge disposition were performed only for patients discharged from the hospital alive.

The composite NIS-SAH outcome measure (NIS-SOM) was evaluated among patients with detailed discharge data available. This validated dichotomous outcome measure defines a poor outcome based on in-hospital mortality, discharge to institutional care (nursing facility, extended care facility, or hospice), or tracheostomy or gastrostomy placement. The NIS-SOM has been shown to have $95 \%$ agreement with the modified Rankin Scale (mRS) when poor outcome was defined as an $\mathrm{mRS}$ score $>3 .{ }^{44}$

\section{Subgroup and Sensitivity Analyses}

Subgroup analyses were performed separately evaluating patients treated with microsurgical clip placement or endovascular embolization. Additionally, a sensitivity analysis was performed accounting for the design of the database. Because the NIS has a limited number of diagnoses it can include from each patient (15 from 2006-2008 and 25 from 2009-2011), it is plausible that antithrombotic medication may be less likely to be encoded in patients with multiple complications using all of the potential diagnosis positions, artifactually making antithrombotic medication associated with superior outcomes. Thus, a sensitivity analysis was performed only including patients who did not use the maximal number of diagnoses. Additionally, in accordance with prior research, ${ }^{20}$ a second sensitivity analysis was performed where patients with a diagnosis code for long-term antiplatelet agent use (V58.63, $\mathrm{n}=39$ ) and a simultaneous diagnosis of anticoagulant and aspirin use $(n=6)$ were also excluded. This analysis was performed because these patients may be at greater risk of hemorrhagic complications from combined antithrombotic medications, without additional antiinflammatory benefits noted from the use of aspirin alone.

\section{Statistical Analysis}

Statistical analyses were performed using Stata 13 (StataCorp, LP) accounting for the survey design of the NIS, with the hospital ID as the sampling unit, the discharge weight as the sampling weight variable, and the NIS stratum as the strata. Baseline characteristics and treatment variables were compared using the chi-square test for categorical variables and the Wilcoxon rank-sum test for nonparametric continuous variables. Hierarchical multivariable logistic regression models were constructed with all predictor variables as covariates, and $\mathrm{C}$ statistics were used to assess the discriminatory capacity of the model. Multivariable linear regression models were used to evaluate length of stay, which was logarithmically transformed due to nonnormal distribution. A p value $<0.05$ was considered statistically significant.

\section{Results \\ Demographics of Study Population}

The study analyzed 11,549 admissions, in which longterm aspirin use was coded in $2.1 \%(\mathrm{n}=245)$ and anticoagulant use in $0.9 \%(n=108)$. Patient and hospital characteristics are compared according to long-term aspirin use in Table 1 and long-term anticoagulation in Table 2. The median age of patients with long-term aspirin use was 62 years (interquartile range [IQR] 54-71 years) and without aspirin use was 54 years (IQR 46-63 years). Patient age, comorbidities, primary payer, and treatment modality used for aneurysm repair varied significantly by long-term aspirin use (Table 1, Fig. 1). Patients with long-term aspirin use had an average of $2.2 \pm 1.4$ comorbidities; nonaspirin users had a mean of $1.7 \pm 1.3$ comorbidities. In the total population, $2.7 \%$ of patients underwent platelet transfusion during the admission $(\mathrm{n}=308)$, and patients with long-term aspirin use were significantly more likely to undergo platelet transfusion $(4.9 \%$ vs $2.6 \%, \mathrm{p}=0.03)$; $2.8 \%$ of patients $(n=322)$ underwent plasma transfusion during the hospital admission, which did not differ significantly according to long-term aspirin use (2.9\% vs $2.8 \%$, $\mathrm{p}=0.95)$.

The median age of patients with long-term anticoagulant use was 67 years (IQR 59-77 years), compared with 54 years (IQR 46-63 years) for non-anticoagulant users. Patients with anticoagulation had an average Elixhauser comorbidity index of $2.4 \pm 1.5$ compared with $1.7 \pm 1.3$ comorbidities among those without anticoagulant use. Patient age, comorbidities, and insurance status all varied significantly by anticoagulant use (Table 2, Fig. 2). Those undergoing anticoagulation did not have a different rate of platelet transfusion ( $1.9 \%$ vs $2.7 \%, \mathrm{p}=0.60)$, but significantly higher rates of plasma transfusion during the hospital admission were observed $(26.9 \%$ vs $2.6 \%, \mathrm{p}<0.001)$.

\section{Outcomes}

The outcomes evaluated were compared based on longterm aspirin use (Table 3, Fig. 3) and long-term anticoagulant use (Table 4, Fig. 2). The median length of hospital stay in the entire population was 16 days (IQR 11-23 days): for patients with long-term aspirin use the median hospital stay was 15 days (IQR 11-21 days), and for non-aspirin users it was 17 days (IQR 12-24 days). In multivariable linear regression, length of hospital stay was significantly shorter (by $12.73 \%$ ) for patients with long-term aspirin use (95\% confidence interval [CI] 5.22\%-20.24\%, p =0.001, $\left.\mathrm{R}^{2}=0.14\right)$. For anticoagulant users, the median hospital stay was 17 days (IQR 12-23 days); for those without anticoagulant use, the median hospital stay was 17 days (IQR 12-24 days). There was a shorter statistical length of stay (by $15.44 \%$ ) in multivariable analyses for patients with long-term anticoagulant use (95\% CI 4.61\%-26.28\%, p = $\left.0.005, \mathrm{R}^{2}=0.13\right)$. 
TABLE 1. Demographics of patients undergoing aneurysm repair after $\mathrm{SAH}$, stratified by long-term aspirin use*

\begin{tabular}{|c|c|c|c|c|}
\hline Variable & $\begin{array}{l}\text { Total Population } \\
\quad(n=11,549)\end{array}$ & $\begin{array}{l}\text { Aspirin Users } \\
\quad(n=245)\end{array}$ & $\begin{array}{l}\text { Non-Aspirin Users } \\
\quad(n=11,304)\end{array}$ & $p$ Value \\
\hline Age (yrs) & & & & $<0.001 \dagger$ \\
\hline $18-45$ & 24.4 & 11.0 & 24.7 & \\
\hline $46-55$ & 30.5 & 17.1 & 30.8 & \\
\hline $56-70$ & 31.9 & 45.3 & 31.6 & \\
\hline$>70$ & 13.2 & 26.5 & 13.0 & \\
\hline Female & 68.4 & 62.0 & 68.5 & $0.03 \dagger$ \\
\hline No. of comorbidities & & & & $<0.001 \dagger$ \\
\hline 0 & 18.2 & 9.0 & 18.4 & \\
\hline 1 & 31.3 & 26.9 & 31.3 & \\
\hline 2 & 26.2 & 29.0 & 26.1 & \\
\hline$\geq 3$ & 24.4 & 35.1 & 24.1 & \\
\hline Insurance status & & & & $<0.001 \dagger$ \\
\hline Private or other & 49.5 & 41.2 & 49.7 & \\
\hline Medicare & 23.4 & 42.9 & 23.0 & \\
\hline Medicaid & 15.6 & 7.8 & 15.7 & \\
\hline Self-payment & 11.5 & 8.2 & 11.6 & \\
\hline Median NIS-SAH SS score (IQR) & $1.2(1.0-8.8)$ & $1.2(1.0-7.6)$ & $1.2(1.0-8.8)$ & $0.03 \dagger$ \\
\hline Intracerebral hemorrhage & 9.7 & 9.8 & 9.7 & 0.95 \\
\hline Cerebral herniation & 4.2 & 3.7 & 4.2 & 0.69 \\
\hline Cerebral edema & 10.0 & 7.8 & 10.1 & 0.24 \\
\hline Microsurgical clipping & 46.1 & 32.7 & 46.4 & $<0.001 \dagger$ \\
\hline Decompressive craniectomy & 1.2 & 0.8 & 1.2 & 0.55 \\
\hline Hospital bed size & & & & 0.15 \\
\hline Small & 3.9 & 2.9 & 3.9 & \\
\hline Medium & 11.1 & 7.8 & 11.2 & \\
\hline Large & 85.0 & 89.4 & 84.9 & \\
\hline Teaching hospital & 89.7 & 89.8 & 89.7 & 0.96 \\
\hline
\end{tabular}

\section{Subgroup Analysis}

Subgroup analyses evaluated patients by treatment modality (Table 3, Fig. 3). The median hospital stay for patients treated with microsurgical clip placement was 17 days (IQR 13-27 days) for those on aspirin and 17 days (IQR 13-25 days) for those not taking aspirin. No significant difference was noted in length of stay (by $1.01 \%$ ) according to aspirin use for patients who underwent microsurgical clip placement $(95 \% \mathrm{CI}-14.44 \%$ to $12.42 \%$, $\mathrm{p}=$ 0.88 ). Among patients treated endovascularly, the median length of stay for aspirin users was 14 days (IQR 10-20 days), and 16 days (IQR 11-24 days) for non-aspirin users. This difference was significantly shorter (by $18.91 \%$; $95 \%$ CI $9.85 \%-27.96 \%, \mathrm{p}<0.001)$.

\section{Sensitivity Analysis}

A sensitivity analysis only evaluated patients who did not use the maximal number of potential diagnoses $(\mathrm{n}=$ 10,514); similar statistical associations were noted between both aspirin and anticoagulation on all outcomes compared with the analysis of the total population (data not shown). A second sensitivity analysis was performed after excluding patients with a diagnosis code of other antiplatelet agents or a combination of aspirin and antico- agulant use $(\mathrm{n}=11,504)$. This analysis showed similar statistical associations for long-term aspirin use with or without other antiplatelet agents or anticoagulants, except that there was only a trend toward lower rates of cardiac complications in aspirin users (6.5\% vs $8.5 \%$, OR $0.63,95 \%$ CI $0.38 \%-1.02 \%, \mathrm{p}=0.06$ ); no difference was observed in rates of venous thromboembolic events $(5.1 \%$ vs $7.8 \%$, OR $0.55,95 \%$ CI $0.23 \%-1.08 \%, \mathrm{p}=0.08$ ), and a lower rate of hematological complications was noted among patients with long-term aspirin use (17.6\% vs $21.2 \%$, OR $0.70,95 \%$ CI $0.49 \%-0.99 \%, \mathrm{p}=0.04)$. Moreover, similar associations between long-term anticoagulant use and outcomes were observed compared with the primary analysis.

\section{Discussion}

Aspirin is an irreversible cyclooxygenase (COX) inhibitor, a family of enzymes that convert arachidonic acid to prostaglandin $\mathrm{H}$. At low doses, aspirin inhibits COX1 in platelets, preventing the formation of thromboxane A2, which is a vasoconstrictor and a thrombogenic factor; at higher doses, aspirin also inhibits COX-2, which is a mediator in inflammatory pathways. ${ }^{8}$ Inflammation may be critical to the formation and rupture of cerebral aneurysms, and thus it is biologically plausible that antiin- 
TABLE 2. Demographics of patients undergoing aneurysm repair after SAH, stratified by long-term anticoagulant use*

\begin{tabular}{|c|c|c|c|c|}
\hline Variable & $\begin{array}{l}\text { Total Population } \\
\qquad(\mathrm{n}=11,549)\end{array}$ & $\begin{array}{l}\text { Anticoagulant Users } \\
\qquad(n=108)\end{array}$ & $\begin{array}{l}\text { Non-Anticoagulant } \\
\text { Users }(n=11,441)\end{array}$ & p Value \\
\hline Age (years) & & & & $<0.001 \dagger$ \\
\hline $18-45$ & 24.4 & 7.4 & 24.6 & \\
\hline $46-55$ & 30.5 & 13.0 & 30.6 & \\
\hline $56-70$ & 31.9 & 35.2 & 31.9 & \\
\hline$>70$ & 13.2 & 44.4 & 13.0 & \\
\hline Female & 68.4 & 69.4 & 68.4 & 0.81 \\
\hline No. of comorbidities & & & & $<0.001 \dagger$ \\
\hline 0 & 18.2 & 7.4 & 18.3 & \\
\hline 1 & 31.3 & 19.4 & 31.4 & \\
\hline 2 & 26.2 & 34.3 & 26.1 & \\
\hline$\geq 3$ & 24.4 & 38.9 & 24.2 & \\
\hline Atrial fibrillation & 5.3 & 43.5 & 4.9 & $<0.001 \dagger$ \\
\hline Insurance status & & & & $<0.001 \dagger$ \\
\hline Private or other & 49.5 & 31.5 & 49.7 & \\
\hline Medicare & 23.4 & 56.5 & 23.1 & \\
\hline Medicaid & 15.6 & 9.3 & 15.6 & \\
\hline Self-payment & 11.5 & 2.8 & 11.6 & \\
\hline Median NIS-SAH SS score (IQR) & $1.2(1.0-8.8)$ & $1.5(1.0-8.8)$ & $1.2(1.0-8.8)$ & 0.45 \\
\hline Intracerebral hemorrhage & 9.7 & 10.2 & 9.7 & 0.86 \\
\hline Cerebral herniation & 4.2 & 1.9 & 4.2 & 0.23 \\
\hline Cerebral edema & 10.0 & 8.3 & 10.0 & 0.56 \\
\hline Microsurgical clipping & 46.1 & 38.9 & 46.2 & 0.13 \\
\hline Decompressive craniectomy & 1.2 & 0.9 & 1.2 & 0.77 \\
\hline Hospital bed size & & & & 0.57 \\
\hline Small & 3.9 & 5.6 & 3.9 & \\
\hline Medium & 11.1 & 9.3 & 11.1 & \\
\hline Large & 85.0 & 85.2 & 85.0 & \\
\hline Teaching hospital & 89.7 & 87.0 & 89.7 & 0.36 \\
\hline
\end{tabular}

* All data are presented as percentages, with the exception of NIS-SAH SS.

$\dagger$ Statistically significant difference by chi-square test for categorical variables or Wilcoxon rank-sum test for continuous variables.

flammatory medications may reduce the risk of rupture of such aneurysms. Inflammatory mediators (including macrophages and $\mathrm{COX}-2$ ) are upregulated in the walls of cerebral aneurysms and have been found to have decreased signal intensity in cerebral aneurysms from patients on aspirin. $^{8}$

Interest in aspirin as an agent for the prevention of $\mathrm{SAH}$ was prompted by a retrospective analysis of patients enrolled in the ISUIA study. In this publication, patients who used aspirin at least 3 times weekly had a lower odds of rupture in multivariate regression models also accounting for age, geographic region, and cigarette smoking. ${ }^{21}$ However, aspirin use may also have been a marker of greater health literacy and superior baseline health, and several potential confounding variables could not be assessed due to data collected. ${ }^{40}$ Likewise, in an analysis of the health improvement network database from the United Kingdom, aspirin use was associated with decreased odds of $\mathrm{SAH}$, with the protective benefit greatest for those with at least 3 years of utilization. ${ }^{19}$ However, data on the association between aspirin and $\mathrm{SAH}$ has been conflicting. A nested case-control study from the German Pharmacoepidemiological Research Database reported that aspirin use was associated with increased odds of $\mathrm{SAH} ;{ }^{18}$ similar results were observed in a population-based case-control study from northern Denmark ${ }^{35}$ and in an analysis of the American Nurses' Health study cohort. ${ }^{28}$ In contrast to the analysis of ISUIA, it is difficult in these population-based studies to determine whether the SAH reported is aneurysmal or nonaneurysmal.

Nevertheless, any potential therapeutic benefit of aspirin must be viewed in the context of antithrombotic properties due to its antiplatelet activity and the theoretical risk of greater hemorrhage if rupture occurs. Data are limited on how prehemorrhage aspirin utilization impacts the outcomes after SAH. In 2004, Touissant et al. reported a retrospective single-institutional series finding that premorbid aspirin use was not associated with differential outcomes, although there was a trend toward a higher rate of rebleeding and a lower rate of permanent disability from vasospasm for aspirin users. ${ }^{39}$ Moreover, in another institutional report, Gross et al. found no impact of aspirin usage on the presentation, short-term, or 1-year outcomes after SAH with aspirin use. ${ }^{20}$ However, these publications were limited by small sample sizes and reduced ability to evaluate differential outcomes by treatment modality.

Aspirin has also been investigated as an agent to reduce the incidence of delayed ischemic neurological deficit af- 
A

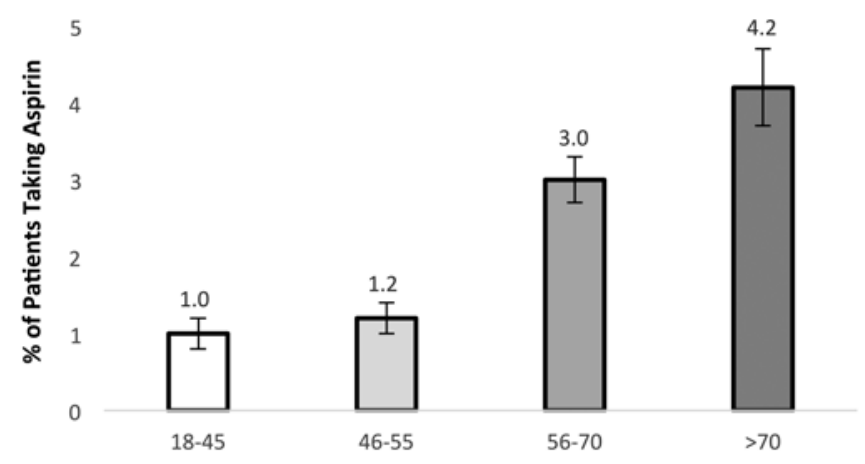

C

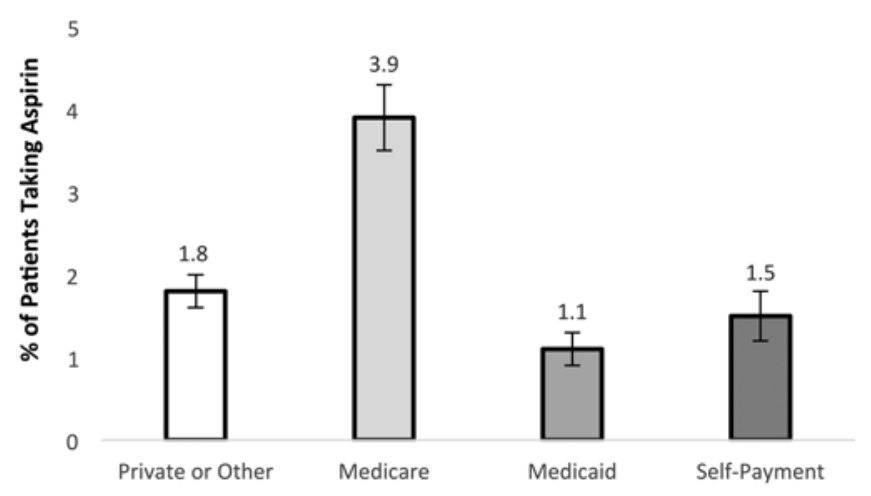

B

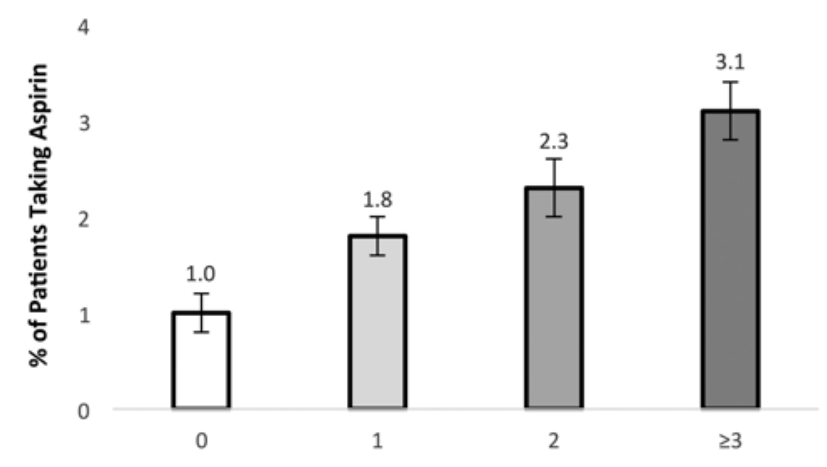

D

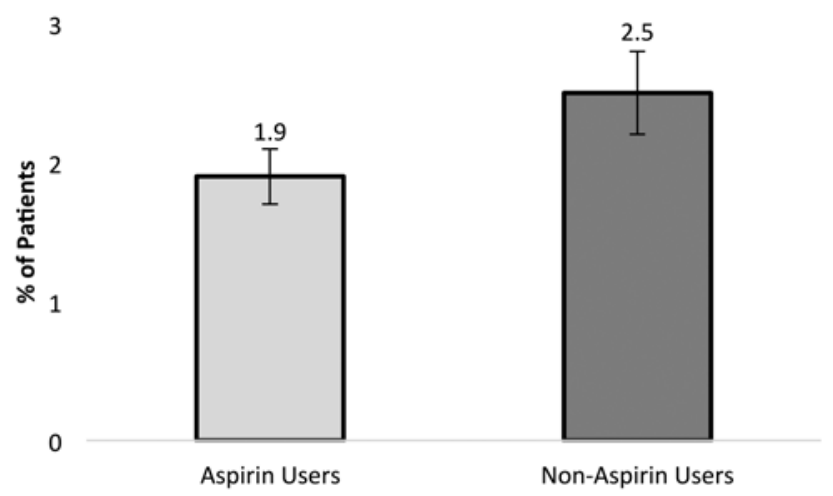

FIG. 1. Variations in the rate of aspirin use (and its associated standard error) according to age (A), number of comorbidities (B), insurance status (C), and female sex (D).

ter SAH. ${ }^{13,27,29,42}$ The pathophysiology of delayed cerebral ischemia is complex, but platelet aggregation and inflammation may be important. Although an initial meta-analysis found a lower pooled rate of delayed cerebral ischemia when aspirin was administered after aneurysm repair, ${ }^{12}$ this benefit was not found in a randomized controlled trial of 161 patients in which $100 \mathrm{mg}$ of aspirin was given postoperatively after microsurgical clipping and continued for 21 days. $^{41}$

The relationship between anticoagulation and the risk of SAH is poorly understood. Several population-based studies have found a significant association between anticoagulant use and $\mathrm{SAH},{ }^{18}$ but determining from these studies which cases are aneurysmal is difficult. A singleinstitution study did not find any cases of rupture in a population of patients with known cerebral aneurysms who were treated conservatively and required anticoagulation; however, many of these aneurysms were less than $7 \mathrm{~mm}$, suggesting a low baseline risk of rupture. ${ }^{38}$ Other institutional studies have found a low rate of good neurological outcomes in patients who sustained rupture while undergoing anticoagulation. ${ }^{33}$

In this study, 11,504 patients from across the US were analyzed to evaluate the impact of long-term aspirin and anticoagulant use on the outcomes after aneurysmal SAH. Similar to prior publications, ${ }^{20}$ aspirin users were found to be older, more likely to be male, and have a higher burden of comorbid disease. Additionally, aspirin users were less likely to undergo aneurysm repair via microsurgical clipping; this may reflect concerns about performing a craniotomy in patients on aspirin, given its limited reversibility. Notably, the severity of SAH as determined by the NISSAH SS score, which has been shown to have a good correlation with Hunt and Hess grade, ${ }^{44}$ was significantly lower in patients with long-term aspirin use. In multivariable regression models that include many important covariates (such as age, comorbidities, severity of SAH, and hospital demographics), in-hospital mortality, total complication rates, and neurological complication rates were not found to differ significantly according to aspirin use. However, long-term aspirin use was associated with significantly lower odds of a cardiac complication or a venous thromboembolic event. Additionally, long-term aspirin use was associated with some superior outcomes, including shorter hospital stay and lower odds of a nonroutine discharge. Although the NIS does not include validated outcomes measures assessing neurological status, discharge disposition may provide an indication of functional independence at the end of the hospitalization. Notably, the statistical benefit to long-term aspirin use was primarily due to superior outcomes in these measures in patients treated endovascularly; likewise, the adjusted odds of a poor outcome were lower for those with long-term aspirin use among patients who underwent coil embolization. Additionally, the direction of the crude rates of mortality and complication rates (although not significantly different) favored aspirin for 
A

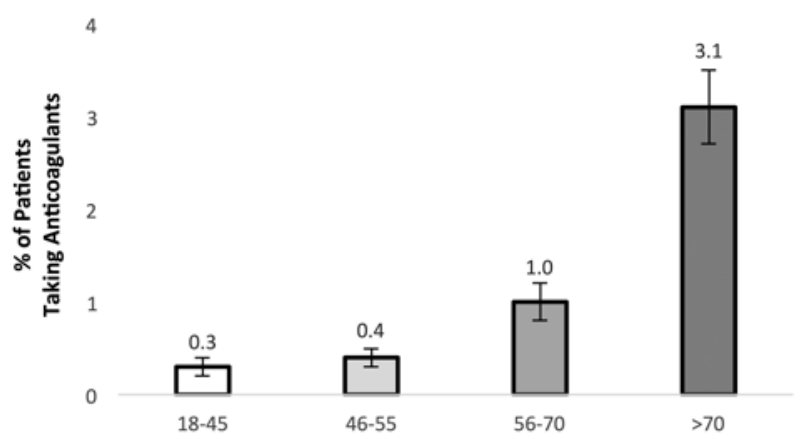

C

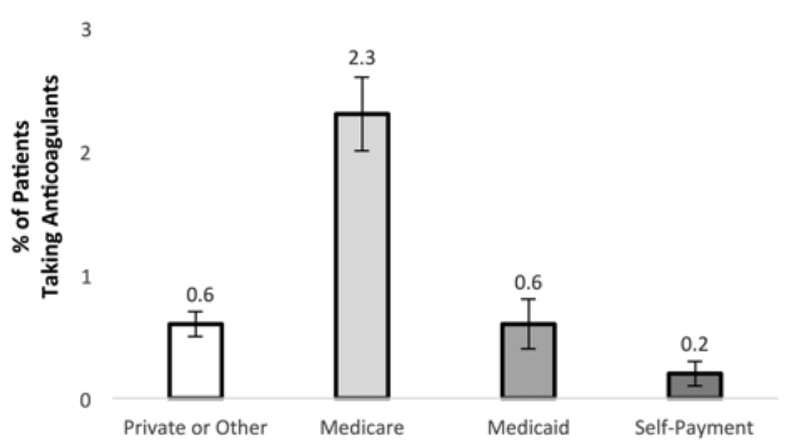

$\mathbf{E}$

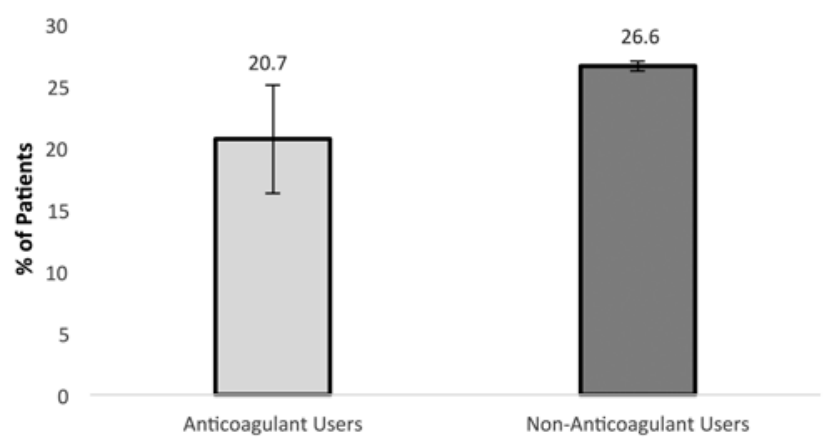

B

\section{Number of Comorbidities}

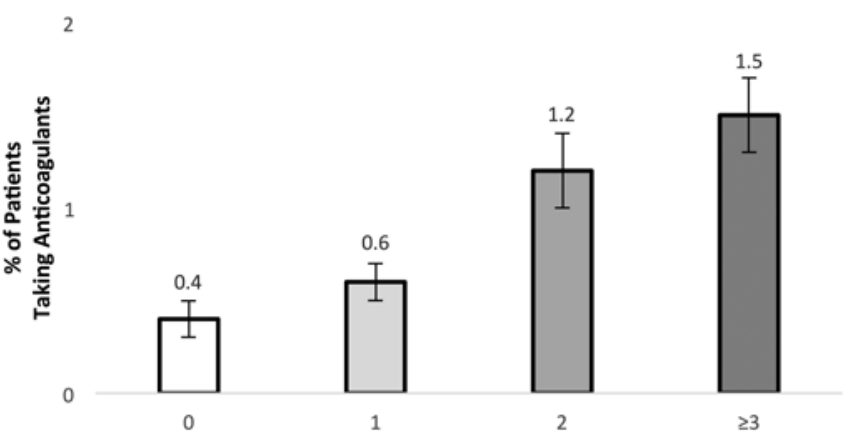

D

Mortality Rates

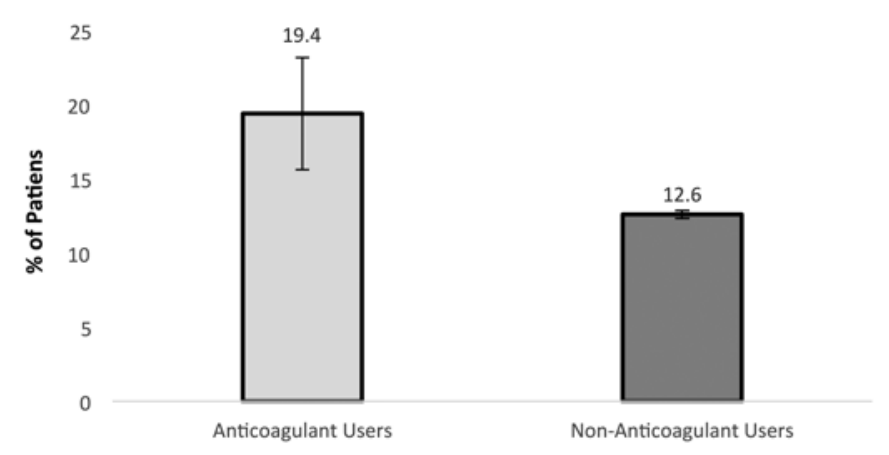

F

Poor Outcome by NIS-SOM

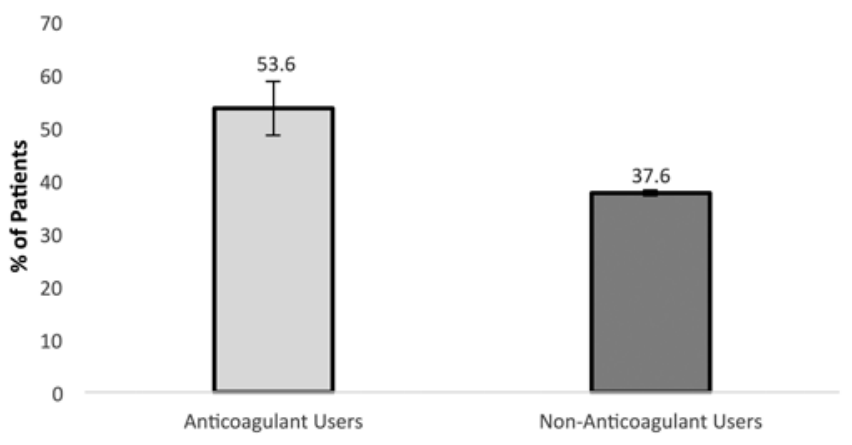

FIG. 2. Differences in anticoagulant use according to age (A), comorbidities (B), and insurance status (C). Comparison by longterm anticoagulant use in the rates of in-hospital mortality (D), the proportion of patients with a length of hospital stay of at least 23 days (E; the upper quartile of the IQR), and poor outcome (F; measured by the NIS-SOM). Data are presented as crude rates with the associated standard errors.

those treated endovascularly and favored non-aspirin users in patients treated surgically.

When analyzing long-term anticoagulant use, differences in age and number of comorbidities were significant and more profound than those noted with aspirin use. The crude rates of in-hospital mortality, neurological complications, nonroutine discharge disposition, and poor outcome were all higher for patients with long-term anticoagulation. However, in multivariable regression models, these outcomes did not differ significantly, suggesting that anticoagulant use may be a marker for inferior baseline health.

This NIS study has notable limitations. One of the key limitations is that it is restricted to patients who underwent aneurysm repair: because the ICD-9-CM code for SAH (430) does not specify if the etiology was aneurysmal, only patients with a diagnosis of SAH who underwent aneurysm repair were included to increase the specificity of the analysis. However, patients with catastrophic hemorrhages leading to prehospital death or precluding aneurysm repair (for example, due to poor grade) could not be evaluated, and it is possible that antithrombotic medication may be associated with such hemorrhages. Moreover, the prevalence of long-term aspirin use in this study (2.1\%) is lower than that reported in prior studies of the SAH population. Toussaint et al. reported $9.5 \%$ of patients had documentation of any aspirin use, although only $5.9 \%$ used aspirin daily or every other day, and $11.7 \%$ of patients in Gross 
TABLE 3. Outcomes after aneurysm repair stratified by long-term aspirin use

\begin{tabular}{|c|c|c|c|c|c|}
\hline \multirow[b]{2}{*}{ Variable } & \multicolumn{2}{|c|}{ Crude Rate } & \multirow[b]{2}{*}{ OR $(95 \% \mathrm{Cl})$} & \multirow[b]{2}{*}{ p Value } & \multirow[b]{2}{*}{ C Statistic } \\
\hline & Aspirin Users & Non-Aspirin Users & & & \\
\hline \multicolumn{6}{|l|}{ Total population } \\
\hline In-hospital mortality & 13.5 & 12.6 & $1.02(0.68-1.52)$ & 0.93 & 0.77 \\
\hline Total complications & 79.6 & 80.0 & $0.80(0.56-1.15)$ & 0.22 & 0.78 \\
\hline Neurological & 32.2 & 32.6 & $1.12(0.84-1.51)$ & 0.44 & 0.60 \\
\hline Cardiac & 6.1 & 8.5 & $0.57(0.36-0.91)$ & $0.02 *$ & 0.67 \\
\hline Venous thromboembolic & 4.9 & 7.8 & $0.53(0.30-0.94)$ & $0.03^{*}$ & 0.63 \\
\hline Hematological & 18.8 & 21.2 & $0.75(0.53-1.05)$ & 0.10 & 0.73 \\
\hline Tracheostomy/gastrostomy & 17.1 & 19.9 & $0.72(0.51-1.02)$ & 0.07 & 0.79 \\
\hline Nonroutine discharge & 54.3 & 55.7 & $0.63(0.48-0.83)$ & $0.001^{*}$ & 0.79 \\
\hline Poor outcome by NIS-SOM & 36.1 & 37.8 & $0.77(0.58-1.03)$ & 0.07 & 0.84 \\
\hline \multicolumn{6}{|l|}{ Microsurgical clipping } \\
\hline In-hospital mortality & 17.5 & 11.9 & $1.34(0.71-2.54)$ & 0.37 & 0.79 \\
\hline Total complications & 83.8 & 79.2 & $1.12(0.61-2.06)$ & 0.72 & 0.77 \\
\hline Neurological & 37.5 & 34.0 & $1.28(0.85-1.90)$ & 0.23 & 0.59 \\
\hline Cardiac & 5.0 & 7.5 & $0.47(0.20-1.09)$ & 0.08 & 0.68 \\
\hline Venous thromboembolic & 1.3 & 8.4 & $0.11(0.02-0.79)$ & $0.03^{*}$ & 0.63 \\
\hline Hematological & 21.3 & 23.8 & $0.72(0.41-1.29)$ & 0.27 & 0.73 \\
\hline Tracheostomy/gastrostomy & 23.5 & 20.3 & $1.00(0.55-1.84)$ & 0.99 & 0.80 \\
\hline Nonroutine discharge & 62.1 & 56.7 & $0.84(0.49-1.44)$ & 0.52 & 0.78 \\
\hline Poor outcome by NIS-SOM & 45.1 & 38.1 & $1.11(0.68-1.81)$ & 0.66 & 0.84 \\
\hline \multicolumn{6}{|l|}{ Endovascular coiling } \\
\hline In-hospital mortality & 11.5 & 13.3 & $0.87(0.53-1.42)$ & 0.57 & 0.76 \\
\hline Total complications & 77.6 & 80.8 & $0.70(0.44-1.12)$ & 0.13 & 0.78 \\
\hline Neurological & 29.7 & 31.3 & $1.07(0.72-1.60)$ & 0.73 & 0.61 \\
\hline Cardiac & 6.7 & 9.3 & $0.63(0.35-1.13)$ & 0.12 & 0.67 \\
\hline Venous thromboembolic & 6.7 & 7.3 & $0.81(0.43-1.53)$ & 0.52 & 0.64 \\
\hline Hematological & 17.6 & 19.0 & $0.76(0.50-1.13)$ & 0.17 & 0.73 \\
\hline Tracheostomy/gastrostomy & 14.1 & 19.6 & $0.61(0.40-0.92)$ & $0.02^{*}$ & 0.79 \\
\hline Nonroutine discharge & 50.7 & 54.9 & $0.55(0.39-0.77)$ & $0.001^{*}$ & 0.79 \\
\hline Poor outcome by NIS-SOM & 31.8 & 37.4 & $0.63(0.42-0.94)$ & $0.03^{*}$ & 0.83 \\
\hline
\end{tabular}

* Statistically significant difference based on hierarchical multivariate logistic regression with all demographic variables as covariates, including age, sex, comorbidities, insurance status, NIS-SAH severity scale, herniation, cerebral edema, treatment modality, and hospital characteristics.

et al.'s series were taking aspirin. The lower rate of aspirin use in our study may limit the generalizability of its findings, as it may reflect an inaccuracy in reporting. Although the NIS-SAH SS is a validated tool for severity adjustment, more conventional severity scales including the Hunt and Hess and Fisher grades were not available, limiting the evaluation of prehemorrhage aspirin use and hemorrhage severity. Dosing and duration of aspirin use could not be evaluated. Additionally, rates of rebleeding could not be assessed, which may be associated with antithrombotic medication. Although ICD-9-CM codes are available for transient ischemic attacks and cerebral infarction, no specific diagnosis code is present for delayed cerebral ischemia, limiting the ability to specifically comment on the relationship between aspirin use and this complication.

However, there are many important advantages to the use of a national database to evaluate the impact of longterm aspirin use on outcomes after SAH. The NIS includes patients from across the US admitted to a range of different hospitals, limiting the bias of academic medical centers or of institutional or regional studies. Additionally, the NIS provides a large sample size with greater statistical power to assess for subtle differences in outcomes after SAH by long-term aspirin use. The data from the NIS is largely in concordance with prior institutional studies, suggesting that long-term aspirin use may not lead to inferior outcomes after SAH; and in fact, aspirin may be independently associated with a mild benefit in outcomes after hemorrhage, particularly for patients treated endovascularly. Nonetheless, the optimal study design, as has been advocated by other authors, would be a prospective, randomized controlled trial evaluating aspirin as an agent for secondary prevention in patients found to have an incidental cerebral aneurysm.?

\section{Conclusions}

In this NIS analysis, 11,504 patients from across the US 
A

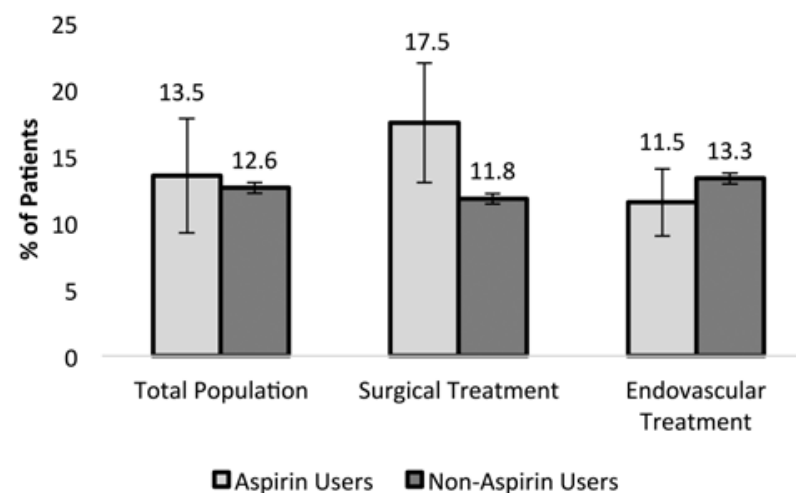

C

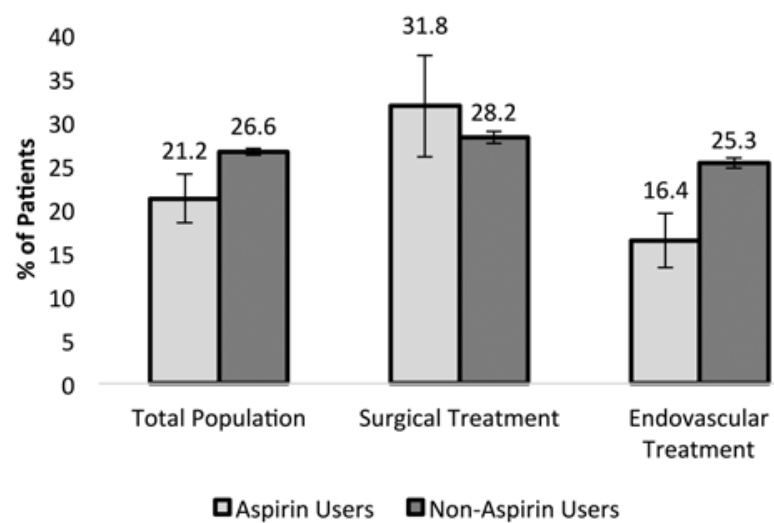

B

Poor Outcome by NIS-SOM

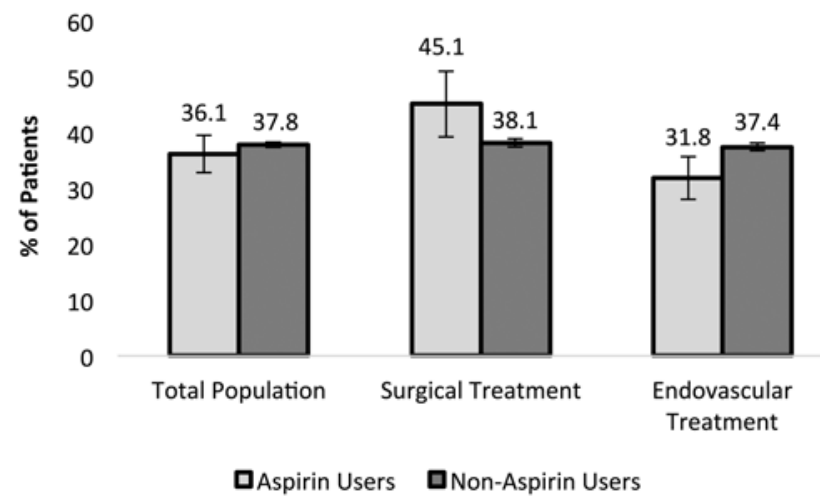

D

Non-routine Discharge Disposition

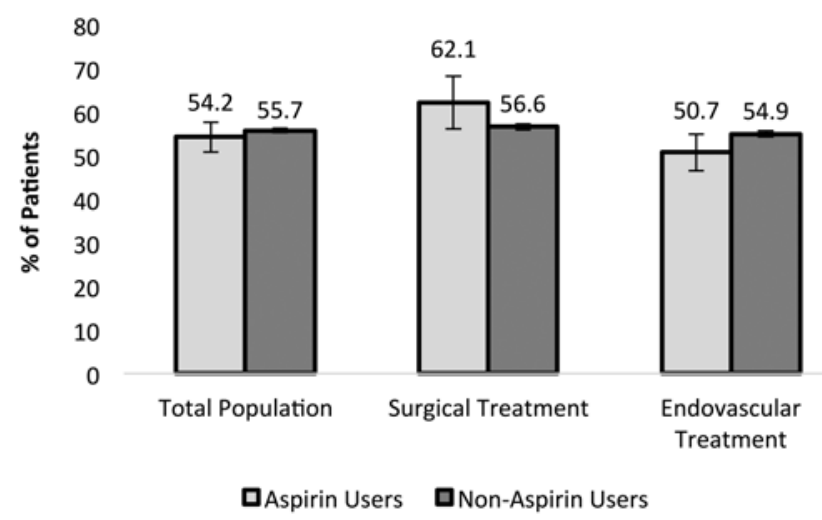

FIG. 3. Comparisons among the total population, in patients treated surgically and in patients treated endovascularly, of the crude rates and the associated standard errors of in-hospital mortality (A), poor outcome (B; defined by the NIS-SOM), the proportion of patients with a length of hospital stay of at least 23 days (C; the upper quartile of the IQR), and discharge disposition (D), according to long-term aspirin use.

were included to evaluate the impact of long-term aspirin and anticoagulant use on the outcomes after aneurysmal SAH. Patients with a history of long-term aspirin use were found to have superior outcomes on some measures, including a significantly shorter hospital stay and lower odds of a nonroutine hospital discharge. Additionally, the odds of a venous thromboembolic event and of a cardiac com- plication were lower in patients with long-term aspirin use. In subgroup analyses, these benefits of aspirin use were primarily noted among patients treated endovascularly. Although the crude rates of mortality and complication rates were higher for patients with long-term anticoagulant usage, these differences were not found to be significant in multivariate analyses.

TABLE 4. The outcomes after aneurysm repair stratified by long-term anticoagulant use

\begin{tabular}{|c|c|c|c|c|c|}
\hline \multirow[b]{2}{*}{ Variable } & \multicolumn{2}{|c|}{ Crude Rate } & \multirow[b]{2}{*}{ OR $(95 \% \mathrm{Cl})$} & \multirow[b]{2}{*}{$p$ Value } & \multirow[b]{2}{*}{ C Statistic } \\
\hline & Anticoagulant Users & Non-Anticoagulant Users & & & \\
\hline In-hospital mortality & 19.4 & 12.6 & $1.36(0.89-2.07)$ & 0.16 & 0.77 \\
\hline Total complications & 85.2 & 80.0 & $0.87(0.52-1.45)$ & 0.58 & 0.78 \\
\hline Neurological & 34.3 & 32.5 & $1.21(0.83-1.78)$ & 0.33 & 0.60 \\
\hline Cardiac & 6.5 & 8.5 & $0.54(0.25-1.16)$ & 0.11 & 0.67 \\
\hline Venous thromboembolic & 13.0 & 8.5 & $1.53(0.88-2.66)$ & 0.13 & 0.63 \\
\hline Hematological & 28.7 & 21.1 & $1.12(0.69-1.81)$ & 0.66 & 0.73 \\
\hline Tracheostomy/gastrostomy & 23.3 & 19.8 & $0.87(0.54-1.42)$ & 0.58 & 0.79 \\
\hline Nonroutine discharge & 75.9 & 55.5 & $1.27(0.73-2.22)$ & 0.39 & 0.79 \\
\hline Poor outcome by NIS-SOM & 53.6 & 37.6 & $1.09(0.69-1.73)$ & 0.72 & 0.84 \\
\hline
\end{tabular}

* Analyses are based on multivariate logistic regression with all demographic variables as covariates, including age, sex, comorbidities, insurance status, NIS-SAH SS, herniation, cerebral edema, treatment modality, and hospital characteristics. 


\section{References}

1. Attenello FJ, Reid P, Wen T, Cen S, Kim-Tenser M, Sanossian $\mathrm{N}$, et al: Evaluation of time to aneurysm treatment following subarachnoid hemorrhage: comparison of patients treated with clipping versus coiling. J Neurointerv Surg [epub ahead of print], 2015

2. Baek H, Jayaraman MV, Karniadakis GE: Wall shear stress and pressure distribution on aneurysms and infundibulae in the posterior communicating artery bifurcation. Ann Biomed Eng 37:2469-2487, 2009

3. Barker FG II, Amin-Hanjani S, Butler WE, Ogilvy CS, Carter BS: In-hospital mortality and morbidity after surgical treatment of unruptured intracranial aneurysms in the United States, 1996-2000: the effect of hospital and surgeon volume. Neurosurgery 52:995-1009, 2003

4. Bekelis K, Missios S, MacKenzie TA, Desai A, Fischer A, Labropoulos N, et al: Predicting inpatient complications from cerebral aneurysm clipping: the Nationwide Inpatient Sample 2005-2009. J Neurosurg 120:591-598, 2014

5. Berger JS, Brown DL, Becker RC: Low-dose aspirin in patients with stable cardiovascular disease: a meta-analysis. Am J Med 121:43-49, 2008

6. Brinjikji W, Lanzino G, Rabinstein AA, Kallmes DF, Cloft HJ: Age-related trends in the treatment and outcomes of ruptured cerebral aneurysms: a study of the Nationwide Inpatient Sample 2001-2009. AJNR Am J Neuroradiol 34:1022-1027, 2013

7. Chalouhi N, Jabbour P, Hasan D, Starke RM: Aspirin for prevention of subarachnoid hemorrhage: the stage is set for a randomized controlled trial. Neurosurgery 74:E147-E148, 2014

8. Chalouhi N, Jabbour P, Starke RM, Hasan DM: Aspirin for prophylaxis against cerebral aneurysm rupture. World Neurosurg 81:e2-e3, 2014

9. Chyatte D, Bruno G, Desai S, Todor DR: Inflammation and intracranial aneurysms. Neurosurgery 45:1137-1147, 1999

10. Claassen J, Bateman BT, Willey JZ, Inati S, Hirsch LJ, Mayer SA, et al: Generalized convulsive status epilepticus after nontraumatic subarachnoid hemorrhage: the Nationwide Inpatient Sample. Neurosurgery 61:60-65, 2007

11. Crowley RW, Yeoh HK, Stukenborg GJ, Ionescu AA, Kassell NF, Dumont AS: Influence of weekend versus weekday hospital admission on mortality following subarachnoid hemorrhage. Clinical article. J Neurosurg 111:60-66, 2009

12. Dorhout Mees SM, Rinkel GJ, Hop JW, Algra A, van Gijn $\mathrm{J}$ : Antiplatelet therapy in aneurysmal subarachnoid hemorrhage: a systematic review. Stroke 34:2285-2289, 2003

13. Dorhout Mees SM, van den Bergh WM, Algra A, Rinkel GJ: Antiplatelet therapy for aneurysmal subarachnoid haemorrhage. Cochrane Database Syst Rev (4):CD006184, 2007

14. Elixhauser A, Steiner C, Harris DR, Coffey RM: Comorbidity measures for use with administrative data. Med Care 36:8-27, 1998

15. Fargen KM, Neal D, Rahman M, Hoh BL: The prevalence of patient safety indicators and hospital-acquired conditions in patients with ruptured cerebral aneurysms: establishing standard performance measures using the Nationwide Inpatient Sample database. J Neurosurg 119:1633-1640, 2013

16. Frösen J, Piippo A, Paetau A, Kangasniemi M, Niemelä M, Hernesniemi J, et al: Growth factor receptor expression and remodeling of saccular cerebral artery aneurysm walls: implications for biological therapy preventing rupture. Neurosurgery 58:534-541, 2006

17. Frösen J, Piippo A, Paetau A, Kangasniemi M, Niemelä M, Hernesniemi J, et al: Remodeling of saccular cerebral artery aneurysm wall is associated with rupture: histological analysis of 24 unruptured and 42 ruptured cases. Stroke 35:22872293, 2004

18. Garbe E, Kreisel SH, Behr S: Risk of subarachnoid hemor- rhage and early case fatality associated with outpatient antithrombotic drug use. Stroke 44:2422-2426, 2013

19. García-Rodríguez LA, Gaist D, Morton J, Cookson C, González-Pérez A: Antithrombotic drugs and risk of hemorrhagic stroke in the general population. Neurology 81:566574, 2013

20. Gross BA, Rosalind Lai PM, Frerichs KU, Du R: Aspirin and aneurysmal subarachnoid hemorrhage. World Neurosurg 82:1127-1130, 2014

21. Hasan DM, Mahaney KB, Brown RD Jr, Meissner I, Piepgras DG, Huston J, et al: Aspirin as a promising agent for decreasing incidence of cerebral aneurysm rupture. Stroke 42:3156-3162, 2011

22. Hennekens CH, Sacks FM, Tonkin A, Jukema JW, Byington RP, Pitt B, et al: Additive benefits of pravastatin and aspirin to decrease risks of cardiovascular disease: randomized and observational comparisons of secondary prevention trials and their meta-analyses. Arch Intern Med 164:40-44, 2004

23. Hoh BL, Chi YY, Lawson MF, Mocco J, Barker FG II: Length of stay and total hospital charges of clipping versus coiling for ruptured and unruptured adult cerebral aneurysms in the Nationwide Inpatient Sample database 2002 to 2006. Stroke 41:337-342, 2010

24. Hoh BL, Kleinhenz DT, Chi YY, Mocco J, Barker FG II: Incidence of ventricular shunt placement for hydrocephalus with clipping versus coiling for ruptured and unruptured cerebral aneurysms in the Nationwide Inpatient Sample database: 2002 to 2007. World Neurosurg 76:548-554, 2011

25. Hoh BL, Nathoo S, Chi YY, Mocco J, Barker FG II: Incidence of seizures or epilepsy after clipping or coiling of ruptured and unruptured cerebral aneurysms in the Nationwide Inpatient Sample database: 2002-2007. Neurosurgery 69:644-650, 2011

26. Hoh BL, Rabinov JD, Pryor JC, Carter BS, Barker FG II: Inhospital morbidity and mortality after endovascular treatment of unruptured intracranial aneurysms in the United States, 1996-2000: effect of hospital and physician volume. AJNR Am J Neuroradiol 24:1409-1420, 2003

27. Hop JW, Rinkel GJ, Algra A, Berkelbach van der Sprenkel JW, van Gijn J: Randomized pilot trial of postoperative aspirin in subarachnoid hemorrhage. Neurology 54:872-878, 2000

28. Iso H, Hennekens CH, Stampfer MJ, Rexrode KM, Colditz GA, Speizer FE, et al: Prospective study of aspirin use and risk of stroke in women. Stroke 30:1764-1771, 1999

29. Juvela S: Aspirin and delayed cerebral ischemia after aneurysmal subarachnoid hemorrhage. J Neurosurg 82:945-952, 1995

30. Kshettry VR, Rosenbaum BP, Seicean A, Kelly ML, Schiltz NK, Weil RJ: Incidence and risk factors associated with in-hospital venous thromboembolism after aneurysmal subarachnoid hemorrhage. J Clin Neurosci 21:282-286, 2014

31. McDonald RJ, Cloft HJ, Kallmes DF: Impact of admission month and hospital teaching status on outcomes in subarachnoid hemorrhage: evidence against the July effect. J Neurosurg 116:157-163, 2012

32. Patrono C, Roth GJ: Aspirin in ischemic cerebrovascular disease. How strong is the case for a different dosing regimen? Stroke 27:756-760, 1996

33. Rinkel GJ, Prins NE, Algra A: Outcome of aneurysmal subarachnoid hemorrhage in patients on anticoagulant treatment. Stroke 28:6-9, 1997

34. Rist PM, Buring JE, Kase CS, Kurth T: Effect of low-dose aspirin on functional outcome from cerebral vascular events in women. Stroke 44:432-436, 2013

35. Schmidt M, Johansen MB, Lash TL, Christiansen CF, Christensen S, Sørensen HT: Antiplatelet drugs and risk of subarachnoid hemorrhage: a population-based case-control study. J Thromb Haemost 8:1468-1474, 2010 
36. Smith GA, Dagostino P, Maltenfort MG, Dumont AS, Ratliff $\mathrm{JK}$ : Geographic variation and regional trends in adoption of endovascular techniques for cerebral aneurysms. J Neurosurg 114:1768-1777, 2011

37. Steinhubl SR, Bhatt DL, Brennan DM, Montalescot G, Hankey GJ, Eikelboom JW, et al: Aspirin to prevent cardiovascular disease: the association of aspirin dose and clopidogrel with thrombosis and bleeding. Ann Intern Med 150:379386,2009

38. Tarlov N, Norbash AM, Nguyen TN: The safety of anticoagulation in patients with intracranial aneurysms. J Neurointerv Surg 5:405-409, 2013

39. Toussaint LG III, Friedman JA, Wijdicks EF, Piepgras DG, Pichelmann MA, McIver JI, et al: Influence of aspirin on outcome following aneurysmal subarachnoid hemorrhage. J Neurosurg 101:921-925, 2004

40. Tymianski M: Aspirin as a promising agent for decreasing incidence of cerebral aneurysm rupture. Stroke 42:30033004, 2011

41. van den Bergh WM, Algra A, Dorhout Mees SM, van Kooten F, Dirven CM, van Gijn J, et al: Randomized controlled trial of acetylsalicylic acid in aneurysmal subarachnoid hemorrhage: the MASH Study. Stroke 37:2326-2330, 2006

42. van den Bergh WM, Kerr RS, Algra A, Rinkel GJ, Molyneux AJ: Effect of antiplatelet therapy for endovascular coiling in aneurysmal subarachnoid hemorrhage. Stroke 40:1969-1972, 2009

43. Veeravagu A, Chen YR, Ludwig C, Rincon F, Maltenfort M, Jallo J, et al: Acute lung injury in patients with subarachnoid hemorrhage: a Nationwide Inpatient Sample study. World Neurosurg 82:e235-e241, 2014

44. Washington CW, Derdeyn CP, Dacey RG Jr, Dhar R, Zipfel GJ: Analysis of subarachnoid hemorrhage using the Nationwide Inpatient Sample: the NIS-SAH Severity Score and Outcome Measure. J Neurosurg 121:482-489, 2014

45. Wilterdink JL, Easton JD: Dipyridamole plus aspirin in cerebrovascular disease. Arch Neurol 56:1087-1092, 1999

\section{Disclosures}

Dr. Aziz-Sultan is a proctor for Covidien and Codman. Dr. Gormley is a proctor for Codman.

\section{Author Contributions}

Conception and design: Du, Dasenbrock. Acquisition of data: Dasenbrock, Yan. Analysis and interpretation of data: Du, Dasenbrock, Gross, Guttieres. Drafting the article: Dasenbrock. Critically revising the article: Du, Dasenbrock, Gross, Gormley, Frerichs, Aziz-Sultan. Reviewed submitted version of manuscript: all authors. Approved the final version of the manuscript on behalf of all authors: Du. Statistical analysis: Dasenbrock. Study supervision: $\mathrm{Du}$.

\section{Correspondence}

Rose Du, Department of Neurosurgery, Brigham and Women's Hospital and Harvard Medical School, 75 Francis St., Boston, MA 02115.email: rdu@partners.org. 